\title{
Muséologies
}

Les cahiers d'études supérieures

\section{Philippe Dubé, directeur du LAMIC, Laboratoire de muséologie et d'ingénierie de la culture}

\section{Alessandra Mariani et Sophie Couture-Samson}

Volume 2, numéro 1, octobre 2007

URI : https://id.erudit.org/iderudit/1033596ar

DOI : https://doi.org/10.7202/1033596ar

Aller au sommaire du numéro

Éditeur(s)

Association Québécoise de Promotion des Recherches Étudiantes en

Muséologie (AQPREM)

ISSN

1718-5181 (imprimé)

1929-7815 (numérique)

Découvrir la revue

Citer ce document

Mariani, A. \& Couture-Samson, S. (2007). Philippe Dubé, directeur du LAMIC,

Laboratoire de muséologie et d'ingénierie de la culture. Muséologies, 2(1),

30-44. https://doi.org/10.7202/1033596ar d'utilisation que vous pouvez consulter en ligne. 


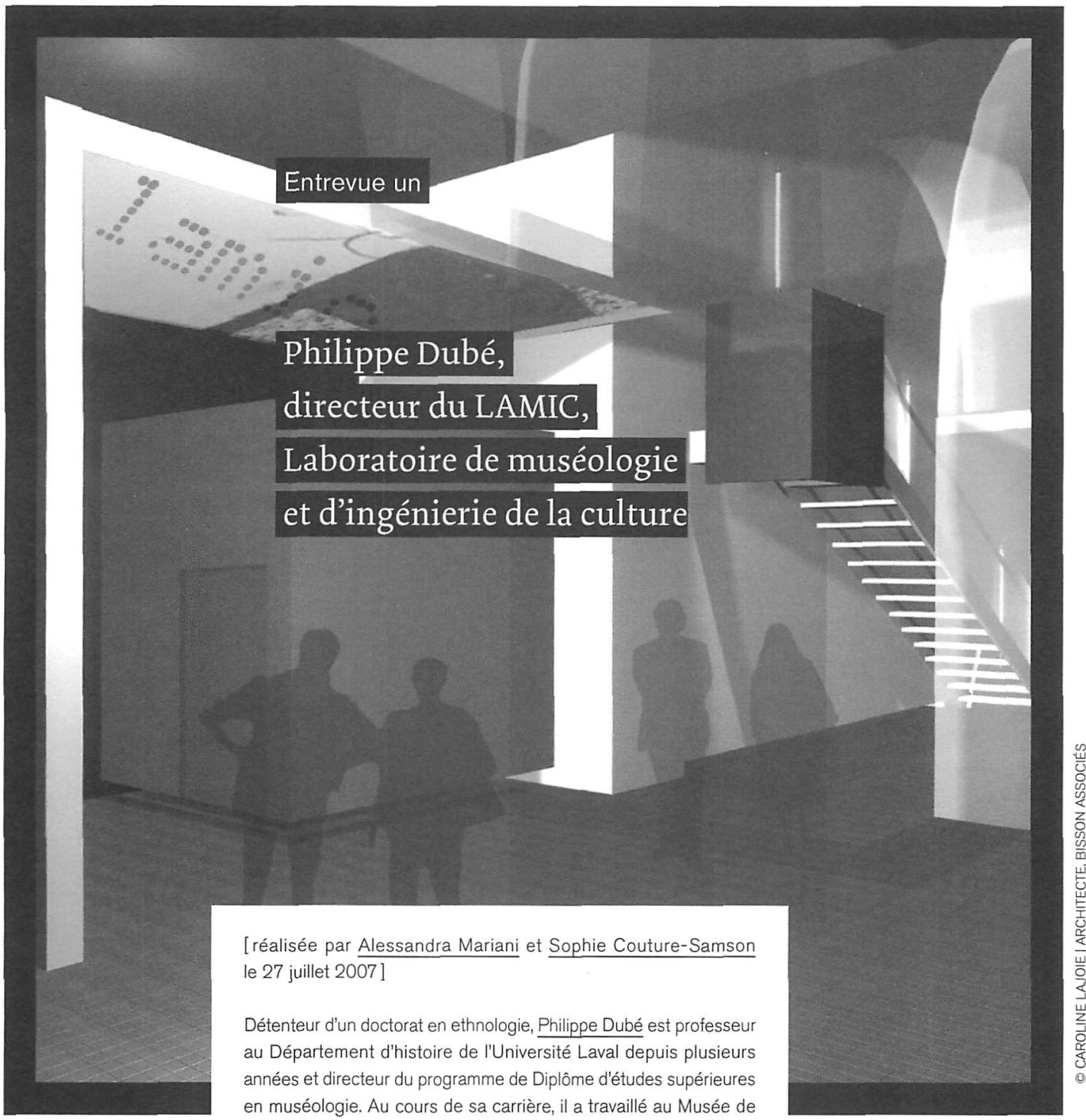
Charlevoix, à Parcs Canada, ainsi qu'au Musée de la civilisation. En mai dernier, il a inauguré le Laboratoire de muséologie et d'ingénierie de la culture (LAMIC), dont il est le directeur. 
A. MARIANI \& S. COUTURE-SAMSON Présentez-nous le Laboratoire de muséologie et d'ingénierie de la culture (LAMIC).

\section{Philippe Dubé}

Il y a bien sûr différentes façons de le présenter. Dans un premier temps, je dirais que ce laboratoire a pour seule prétention d'offrir une infrastructure de recherche expérimentale à celles et à ceux que la question du musée intéresse. Donc, il faut écarter tout de suitel'idée d'un centre de recherche, parce qu'il s'agit plutôt d'une plateforme au service des chercheurs. Nous avons alors centrél'idée maîtresse de ce projet surl'étude des modalités de transmission de la culture en contexte muséal, parce qu'en fait tout gravite autour d'une question assez grave, que nous appelons problématique, en termes de recherche. Cette problématique, cette question fondatrice, se formule de la façon suivante : dans quelle mesure le musée est-il capable de transmettre de la culture, et selon quelles modalités?

Le LAMIC permettra enfin de venir vérifier, mesurer, valider la capacité du musée à transmettre : c'est ce qui nous intéresse au premier chef. C'est vraiment le point de départ, la question centrale et, au fond, toute l'articulation de l'infrastructure a été pensée en ces termes, autour de cette question-là.

Au-delà de la recherche expérimentale, il faut aussi englober la "recherche appliquée", parce que les technologies émergentes nous permettent aujourd'hui de faire des bribes de recherche sur une dimension très spécifique, en lien évidemment avec une question plus large, plus fondamentale, comme la transmission de la culture. Elles nous donnent la possibilité d'aller outre, de ne pas nous confiner au seul croisement image-son-objet d'un contexte donné, par exemple, mais d'aller même au-delà de la recherche fondamentale et de parler éventuellement à partir de la périphérie, des marges. Le LAMIC s'est orchestré intellectuellement autour d'un concept, mais il fallait tout de même en arriver à une définition simple, de base s'il en est, appliquée et applicable du musée. De là, nous avons tiré trois mots qui sont venus définir le projet dans son ensemble : OBJET, VISITEUR et ESPACE. Il faut d'abord comprendre le mot OBJET en son sens le plus large possible. Sans entrer dans le débat du patrimoine matériel et/ou immatériel - parce qu'il ne s'agit pas ici de cela-, il faut plutôt l'entendre comme artefact ou "mentefact " au sens de document culturel, ou encore comme tout ce qui est créé par l'homme et qui peut éventuellement intéresser le musée. Voilà ce qui entre sous le vocable OBJET. Ce qu'il faut aussi savoir, c'est que nous nous sommes donné les moyens d'explorer, de manière réelle et virtuelle, sur les deux registres, l'OBJET, le VISITEUR et l'ESPACE. 
Nous nous retrouvons donc dans l'ordre du réel, de la réalité, et dans celui de la simulation, de la virtualité. La potentialité sémantique du mot simulation, dans le croisement de ces trois eléments fondateurs du musée, nous a beaucoup aidés à structurer le programme de recherche et, de façon plus pressante, le programme architectural, puisque trois zones vont s'occuper spécifiquement de chacun de ces éléments. Le projet architectural du LAMIC s'est donc développé dans une sorte de triade où chaque élément est interdépendant l'un de l'autre. Enfin, l'elément d'aboutissement de cette triade est évidemment l'espace muséal que nous avons baptisé EXPOLAB, parce que c'est la rencontre de l'objet et du visiteur dans un espace donné qui crée tout le sens de ce que nous appelons encore aujourd'hui le musée.

A. MARIANi \& S. CoUture-SAMSON Préconisez-vous vraiment l'espace? Croyez-vous que le fait d'être justement dans une période de I'histoire où l'authenticité de l'objet n'a souvent plus la même valeur, qu'il puisse être remplacé ou carrément subordonné à d'autres fonctions que celle de témoin, puisse valider ce choix? Est-ce parce que le sens ne découle plus directement de l'objet?

\section{Philippe Dubé}

Sans aller jusque-là, il faut savoir que les mots ici ont un sens fort. OBJET parce que nous n'avons pas trouvé mieux. Il faut aussi le comprendre comme étant la production du sujet qui est l'Homme; donc c'est uniquement surces points de base que nous nous sommes entendus surle terme OBJET. On peut constater aussi que la définition de ce terme est large : la chanson, qui est une production de l'homme, va nous intéresser autant que l'objet matériel recueilli par l'ethnologue. Je dirais que, dans ce contexte, ce n'est pas l'espace qui est directement préconisé. Le terme VISITEUR ici fait référence à la vue (visu), au premier sens du terme, puis à vision, visualisation, visuel et visite. Ces concepts ont déterminé et ont permis d'elaborer au sein du laboratoire une clinique de visualisation en croisant plusieurs technologies dans ce domaine très particulier. L'espace étant le volume dans lequel le visiteur et l'objet se rencontrent, c'est peut-être l'endroit où le sens "expositionnel " se crée, mais cela reste à analyser afin de mieux comprendre les enjeux de la mise en espace muséale. Pour le moment, on énonce des hypothèses, mais il reste évidemment à les vérifier. C'est une des raisons de la création du LAMIC. Toutefois, l'espace reste très important à nos yeux (et en cela les géomaticiens spécialistes de la mesure de l'espace nous le confirment tous les jours), puisque nous entretenons une relation particulière avec lui, surtout au musée. C'est aussi l'endroit où la rencontre visiteur-objet se fait d'une manière toute spéciale et le volume porteur de cette rencontre a aussi, fort probablement, une influence surla relation du visiteur avec l'objet. En somme, vous l'aurez compris, c'est le croisement de ces trois entités fondamentales du musée qui intéresse le LAMIC. 
A. MARIANI \& S. COUTURE-SAMSON Mais c'est pourtant clair que cela va toutefois au-delà de la vue : lorsqu'on visite vos laboratoires, les dimensions haptique, auditive et kinésique sont tout aussi présentes, non?

Philippe DUbÉ

Oui, sauf que ce que nous disons et que nous présumons en fait est que la vue en musée est tout de même l'organe par lequel transite de façon prioritaire l'information à livrer, à partager. Elle est ensuite refilée ailleurs dans le corps du visiteur où elle est secondée, prolongée par les autres sens. Mais, pour nous, la vue reste le "capteur principal » de l'expérience de visite (visu) et, de ce point de vue, les mots de notre triade OBJET/ VISITEUR/ESPACE ont étébien choisis.Je veux souligner dans ce même ordre d'idées quel'espace, pour le LAMIC, est fondamental, puisqu'il ne désigne pas seulement une salle d'exposition, mais bien tout ce qui peut se référer au concept de musée, intérieur et extérieur / intra et extra muros, que nous appelons dans notre jargon "muséalité». C'est un peu à l'image des archéologues qui travaillent en archéomatique, qui ont leur espace de fouilles et, à l'aide d'outils de captation, arrivent à reconstituer et à mieux comprendre les données qu'ils ont cumulées de leur terrain. Le LAMIC se propose de travailler de son côté en une sorte de "muséomatique". En effet, nous désirons créer un croisement entre la muséologie et la géomatique afin de mieux comprendre l'espace-musée. Comment? Par la saisie quel'on a del'espace et de ses mesures, soit dans la perception, dans les sensations quel'on peut en avoir, à travers les éléments réceptifs que tout visiteur capte de façon consciente ou inconsciente et qui, nécessairement, influencent son parcours; par la saisie, l'appréhension, la comprêhension de ce même espace, ainsi que par l'analyse des sensations de confort et d'inconfort qu'il peut ressentir. Toutes ces nuances sont mesurables par les sciences de la géomatique cognitive et il devient, par exemple, possible aujourd'hui de comprendre par des données extrêmement précises où survient l'inconfort ("museum fatigue») dansl'expérience de visite. Nous ne sommes plus dansl'ordre de l'impression, ni de la spéculation, mais plutôt dans celui de données quantifiables et vérifiables.

Enplusdufait queles troistermes OBJET/ VISITEUR / ESPACE aient étéles eléments fondateurs du LAMIC, je voudrais mentionner le second volet subsidiaire au premier, soit celui de la muséalité sur laquelle nous nous sommes mis d'accord lors de la définition de notre credo de recherche en tant qu'équipe (Groupe d'étude de la muséalité). La muséalité peut en effet se définir comme réunissant les qualités propres qui sont associées au musée. Dès qu'entre en considération le fait qu'il y a une trace à conserver, dès que la transmission devient une préoccupation réelle, la muséalité, comme principe de base, $y$ est convoquée au premier plan. La mémoire qui, malheureusement, a été un terme galvaudé ces dernières années 
par les historiens et leurs thuriféraires, aurait pu aussi faire partie de cette énonciation. Toutefois, nous avons choisi d'évoquer plutôt la "transmission" pour nous sortir justement du paradigme mémoriel et ne pas nous confiner exclusivement à l'espace muséal en tant que tel (intra muros s'entend). Nous voulons, avec le LAMIC, clairement agréger un maximum d'intérêts scientifiques sur la question de l'expérience muséale au sens plein du terme. À un niveau plus personnel, l'ethnologue que je suis est convaincu $d u$ fait que la question $d u$ musée - bien qu'étroitement liée à la civilisation occidentale - exclut par sa définition encore certains groupes de l'humanité. Dans la mesure où la culture se transmet partout, et que l'on a tendance à penser que le musée y joue un rôle particulier, comment expliquer le fait que toutes ces cultures qui n'ont pas connu le musée se sont tout de même transmises à travers les siècles, voire des millénaires, sans lui? Pensons à la culture inuit par exemple, qui induit le terme "musée" par la notion de maison de transmission-l'endroit où se passel'héritage ou encore le musée au degré zéro, là où l'objet malgré tout joue un rôle de déclencheur dans le mode de passation culturelle. Ce simple fait ethnologique permet de réfléchir sur le contexte muséal au sens large du terme, c'est-àdire sur cet acte de transmission par ailleurs qu'embrasse parfaitement bien la nouvelle muséologie : saisir en somme ce qui éch appe au musée classique ou traditionnel.
A. MARIANI $\&$ S. COUTURE-SAMSON Comment en êtes-vous arrivé à concevoir ce laboratoire?

Philippe Dubé

Le laboratoire émerge d'une crise grave, je dirais même très grave. Je me suis rendu compte de cet état de crise en 2001-2002 où, lecture après lecture (je n'écris pas beaucoup, mais je lis continuellement, et c'est une discipline que je m'impose depuis plus de vingt ans), se révélait un piétinement, une sorte de stagnation intellectuelle (impasse), en ce sens quel'on revenait constamment sur les mêmes propos, avec les mêmes idées. J'étais mal à l'aise face à mes étudiants, ici à l'Université Laval, parce que je n'arrivais plus à les stimuler pour travailler dans les musées : au contraire, je les en eloignais malgré moi. Je ne savais plus à quoi au juste mon enseignement participait, tant la distance critique vis-à-vis de mon objet d'étude était devenue grande. Tout ce temps, j'avais l'impression d'être un chercheur aveugle qui tentait de débusquer des connaissances qui nous étaient jusqu'alors inconnues, et qui nous permettraient éventuellement de mieux cheminer dans cette aventure muséale.

Je ne porte pas ici de jugement de valeur sur les musées eux-mêmes, mais nous n'avons qu'à penser à tous ces lieux communs que nous retrouvons ici et là et qui nous éloignent de ce que doit être le musée de façon absolue. Par exemple, très souvent, ce qui nous est 
présenté en exposition est ce que le public attend. À mon avis, le musée est avant tout un lieu d'émerveillement, d'étonnement, mais si nous nous limitons à ne présenter que ce qui est convenu, il me semble que nous ne sommes plus au bon endroit et que, de surcroit, nous n'utilisons pasle bon médium pourinstruireles publics, les éveiller à l'Autre. C'est au fond venir valider, donner du poids et mettre beaucoup d'accent sur des faits qui sont malheureusement reconnus pour être des lieux communs dans la culture générale. Je veux aussi faire une distinction importante : c'est au sein même de la discipline scientifique que ces effets de banalisation étaient aussi dérangeants, autant qu'à l'échelle de la programmation des musées. À partir de ce moment-là, soitje démissionnais, ce qui n'était pas raisonnable, soit je tentais de trouver une autre avenue, une solution à ce cul-de-sac. C'est à ce moment précis que l'idée de la mesure scientifique est entrée en jeu.

Mon problème est qu'au départ je ne crois pas que la muséologie soit une science. C'est un lieu certes de discours, parfois même savants, mais puisque nous devons nous définir comme scientifiques, aussi bien nous associer directement aux sciences exactes pour mieux baliser ce que nous voulons faire plus précisément. À ce problème d'ordre épistémologique, nous avons essayé d'esquisser un paradigme qui pourrait éventuellement penser le musée dans sa totalité, dans sa globalité. C'est lors d'un voyage en Arménie et en Russie, où j'allais rejoindre Étienne Berthold - étudiant russophone qui alors documentait le propos de son essai en muséologie -, que j'ai été confronté à la question de la transformation, d'une sorte de mutation de la muséologie dans un champ beaucoup plus vaste qu'on appelle là-bas la «culturologie ${ }^{[1] » .}$

\section{[1]}

"Le concept de 'culturologie' (kul'turologija) traduit l'une des évolutions profondes du monde intellectuel russe de ces dernières années. II accompagne le vaste mouvement de renaissance des interrogations à caractère global sur le devenir des civilisations de Samuel P. Huntington et d'un certain comparatisme inter-culturel. Ce terme désigne d'abord une réalité universitaire qui émerge dès le tournant politique de 1991 et qui ne se limite pas à l'actuelle Fédération russe. Elle se constitue rapidement comme une discipline à part entière et s'inscrit dans le paysage des connaissances au même titre que la sociologie, les mathématiques ou la linguistique. Elle redessine le contenu de certains enseignements ainsi que l'allure générale des publications plus ou moins spécialisées [...] la culturologie désigne plus fondamentalement une sorte de philosophie globale qui poursuit l'idéal d'une synthèse de toutes les activités culturelles. À la différence du cultural turn américain et des Kulturwissenschaften allemandes, qui s'intéressent prioritairement aux micropratiques sociales, elle se détermine elle-même comme une science de l'identité qui privilégie le 'paradigme civilisationnel'. En rupture complète avec les précédentes décennies, elle revalorise par exemple la distinction entre les sciences sociales et les humanités classiques pour mieux redéfinir l'unité culturelle. " (REMAUD, Olivier. Russie : La * culturologie ", nouvelle science des civilisations dans la République des idées. <http://www.repid. com/spip.php?article 135> (consulté le 3 août 2007). 
Ce fut alors le choc de la véritable découverte d'un paradigme englobant qui permettait finalement de situer le musée parmi beaucoup d'autres acteurs qui "jouent" un rôle sur la scène de la culture.

Au préalable, lors de discussions avec mes étudiants au moment de la "crise", je regardais avec envie du côté des scientifiques - des sciences de la nature plus particulièrementpour me rendre compte que ces derniers possèdent depuis longtemps un concept englobant qui rallie chacun des champs qui leur sont spécifiques et qu'ils nomment "écologie». Aujourd'hui, personne ne s'aventurerait à traiter d'une question très pointue, très précise en matière de nature, sans se référer explicitement à cette science du système de la nature qui permet de comprendre à une plus petite échelle ce qui peut se passer dans une plus grande, en se situant par rapport à l'environnement. Si nous ramenions cette démarche globalisante à notre réalité, nous sommes en effet spécialistes d'une toute petite chose dans le monde de la culture qui s'appelle musée, et s'y pencher pour toujours gratter sur les mêmes sujets ne donne pas, à mon sens, des résultats très probants. Par ailleurs, se rallier à un plus large paradigme comme la culturologie nous permettrait d'accéder à un autre niveau de lecture et de penser le musée dans la culture dans un sens beaucoup plus large. Cette prise de conscience m'aura permis de sortir enfin du cul-de-sac intellectuel dans lequel je m'étais enfermé et m'a aussi permis de comprendre qu'il était possible de travailler de façon plus large, plus globale en muséologie, d'où l'idée d'un laboratoire de muséologie et d'ingénierie de la culture, la culture étant notre point de départ et notre point d'aboutissement évidemment.

A. MARIANI \& S. COUTURE-SAMSON Votre formation, votre expérience universitaire et professionnelle ont certainement eu un poids sur l'idéalisation puis la mise en forme d'un tel endroit. Quels sont les moments clé de ce cheminement?

PhilipPe DUBÉ

Quand j'ai commencé mes études universitaires, je me suis rendu compte assez rapidement que les choses intellectuelles pouvaient facilement tourner à vide et c'est alors que j'ai ressenti le besoin de m'ancrer dans une sphère concrète. Je m'explique : le fait qu'une grande part de la société, de la collectivité, soit exclue des bénéfices financiers, sociaux, culturels, etc. me dérangeait énormément et continue de m'indisposer encore. Je me réclame d'une pensée d'inspiration socialiste-libertaire (un non-sens selon les marxistes orthodoxes) et ma base de justice sociale est toujours restée vive. Partant de là, je n'ai jamais su dépasser ce stade de conscience des autres et mes lectures sont presque toujours orientées par ce j'ai carrément quitté l'université pour aller à l'école de menuiserie-charpenterie et je suis devenu menuisier-charpentier en bonne et due forme 
(carte de qualification à l'appui), parce que c'était une manière d'enraciner mes actions dans le réel qui me semblait essentielle.

Ce que j'entendais par le réel était, à ce moment de ma vie, représenté et incarné d'une certaine manière par la classe ouvrière : je voulais donc participer à ce but de rallier le plus grand nombre aux bénéfices qu'une société pouvait produire. Cela m'a ramené un peu plus tard en ethnologie avec, cette fois, la ferme conviction de travailler dans ce sens social et communautaire et ce, sans aucune prétention. L'ethnologie a été pour moi le ferment de tout ce qui a suivi, unissant parfaitement le social (concret) et l'intellectuel (abstrait). C'est cette discipline qui m'a inculqué que tout être humain, toute collectivité, toute communauté, avec les moyens dont elle dispose, crée de la culture. À ce stade, on n'est plus dans l'ordre des moyens, on se trouve dans l'état d'être de l'humain (comprendre l'ontologique). Sans culture, l'être humain est condamné à mourir (Edgar Morin) ${ }^{[21}$ Cette croyance m'a amené vers le musée et, dans mon cas, il s'est agi du Musée régional de Charlevoix.

\section{[2]}

Edgar Morin écrit précisément ceci : "L'homme est un être culturel par nature parce qu'il est naturel par culture". In. Le paradigme perdu : la nature humaine. Paris : Seuil, 1973, p. 101.
Au départ, c'était tout à fait à l'opposé de ce que je voulais faire, parce que je devais m'intéresser à la villégiature plutôt bourgeoise du lieu. Mais cette expérience professionnelle m'a convaincu d'une chose d'un tout autre ordre, et je reprends ici l'adage bien connu : c'est dans les petits pots que se trouvent les meilleurs onguents. Il s'agit de l'idée qu'un petit musée est un lieu parfaitement "sur mesure" pour faire de la transmission. À cet endroit, j'ai vécu et appris des choses extraordinaires (entre autres, "Less is more", "Think global, act local »...). Conservateur et commissaire d'expositions, j'ai été à plusieurs reprises témoin de l'efficacité de ces moyens de transmission par le simple témoignage de visiteurs.

Par la suite, j'ai travaillé comme conservateur à Parcs Canada, une organisation centrée sur la question de l'interprétation de notre histoire nationale et l'importance $d u$ visiteur par un accueil approprié, c'est-à-dire une compréhension claire $d u$ devoir $d u$ parc ou du site (mission) à livrer une grille de lecture pour accompagner l'émerveillement et l'étonnement de ses visiteurs. Parce que si l'on crée un parc, c'est qu'il s'y trouve des eléments de la nature ou de la culture considérés comme exceptionnels. On pourrait bien sûr facilement clôturer ces endroits, les muséifier en somme, et laisser les gens libres d'y circuler. Mais Parcs Canada a choisi d'accompagner 
la découverte du visiteur par des techniques et des programmes bien particuliers qui relèvent de l'interprétation de la grande école américaine. Cette deuxième expérience de travail a été pour moi vraiment marquante.

Ma dernière expérience en tant que professionnel de musée s'est passée au Musée de la civilisation, ici même à Québec, où j'ai travaillé à la mise en place de l'exposition inaugurale "Mémoires" à titre de chargé de projet. J'ai retenu de cette expérience la constante référence aux autres. À titre de chef de cette grande équipe réunie pour réaliser une exposition permanente en 18 mois (d'une durée de vie de 15 ans), je devais sans cesse rappeler que cette mise en espace devait être accessible au plus grand nombre, sans sous-évaluer les connaissances des visiteurs. La phrase que je répétais inlassablement à l'équipe était la suivante : "Ma tante Janine est une femme intelligente, qui ne possède certes pas votre savoir, mais qui a le droit de comprendre et d'apprécier. "Quitteà en paraître ridicule, cette phrase m'aura permis de leur rappeler de bien marquer une frontière qui devait être respectée entre le connaisseur et celui ou celle qui veut connaître. La présence de Roland Arpin à la direction de ce musée au moment de sa conception a largement contribué à ce que le musée devienne un endroit s'adressant à l'intelligence $d u$ plus grand nombre. Je crois fermement que ces expériences professionnelles, liées au respect des publics, m'ont permis de mieux penser l'infrastructure de recherche qu'est le LAMIC.

A. Mariani \& S. Couture-Samson

À qui le LAMIC s'adresse-t-il vraiment?

Philippe Dubé

Fondamentalement, notrepréoccupation est de savoirsilemuséeremplitpleinementsafonction dans la société d'aujourd'hui. Je pense qu'au volet de la transmission à long terme, le musée a largement sa place (conserver le patrimoine pour le transmettre aux générations futures). C'est dans la transmission à court terme qu'il mérite d'être examiné de façon plus précise. À quoi une infrastructure comme celle-ci peut-elle être utile dans un pareil contexte? Encréant le LAMIC, nous nous sommes engagés dans deux voies principalement.

La première a été de nous associer à des institutions muséales. Nous en avons ciblé trois, une à Montréal, une à Québec et une autre en région, à Saguenay précisément. Ce choix obéissait à la fois à une logique géographique, à une logique thématique et à une logique de bonne entente, puisque que nous devions obtenir l'accord et même l'enthousiasme des institutions muséales impliquées. Nous avons d'abord choisi le Musée d'art contemporain de Montréal, parce que c'est un lieu qui ne se dément pas sur les plans de l'innovation et de l'actualisation des contenus culturels 
émergeants; c'est un lieu qui est aussi préoccupé par le partage avec le plus grand nombre, par la transmission du savoir malgré la difficulté de certains sujets abordés. En ce qui concerne le Musée de la civilisation du Québec, il allait de soi que nous devions nous associer à cette institution puisqu'il s'agit d'un musée de société, exemplaire à bien des égards, qui s'inscrit parfaitement dans nos recherches. Enfin, nous avons choisi la Pulperie de Chicoutimi car il s'agit d'un site industriel, situédans une région dans laquelle le GRAMUL (Groupe en Recherche-Action en muséologie à l'Université Laval) s'est beaucoup investi, et parce qu'il y a, à proximité, un patrimoine naturel et culturel qui nous intéresse particulièrement, entre autres celuides Amérindiens avec le musée de Mashteuiatsh. En nous associant avec ces trois institutions, nous avons voulu ouvrir le LAMIC, montrer qu'il ne s'agit pas d'un club universitaire fermé. Le LAMIC demande d'ailleurs à ses associés de jouer un rôle d'ouverture et d'entremise et de rallier ainsi un maximum d'autres musées.

Cependant, l'association avec ces partenaires ne signifie pas qu'ils soient les seuls avec lesquels le laboratoire entretient des relations étroites. Nous avons entre autres une belle complicité avec le Centre d'histoire de Montréal, l'Écomusée du Fier Monde, le Musée du Fjord, le Musée des Ursulines de Québec, le Musée $\mathrm{du}$ Bas-Saint-Laurent et avec bien d'autres musées qui n'ont pas les moyens de ces grandes institutions, mais qui, par ailleurs, partagent certains de nos intérêts. Les trois institutions ciblées sont donc des institutions phares au sein du réseau des musées québécois et elles permettront au LAMIC de se rallier à d'autres en assurant un relais avec le plus grand nombre.

La seconde voie a été de nous associer directement avec des firmes privées qui sont déjà compétentes dans leur domaine. Au volet de la numérisation ou captation $3 D$, nous avons choisi $M C G{ }_{3} D$ et à celui de la réalité augmentée, notre choix s'est arrêtésurÉmotion Technologies. Il s'agit de deux firmes de Québec. Nous avons également des liens particuliers avec la jeune firme Polyrix, qui œuvre dans le théatre $3 D$, pour qui le LAMIC pourrait servir de tremplin (lire "spin off"). Ces deux voies d'orientation nous ont permis de nous associer à des partenaires directement intéressés par ce que nous faisons dans une perspective de partenariat public-privé (PPP) Donc, pour répondre à la question à qui nous adressonsnous? À l'ensemble de la communauté muséale, à travers des représentants de deux univers que nous essayons de concilier à partir de l'université : celui des institutions muséales et celui des firmes muséographiques qui travaillent pour ces institutions, afin de pouvoir être un lieu de rencontre neutre en dehors d'une stricte relation d'affaires. Habituellement, un musée embauche une firme muséographique 


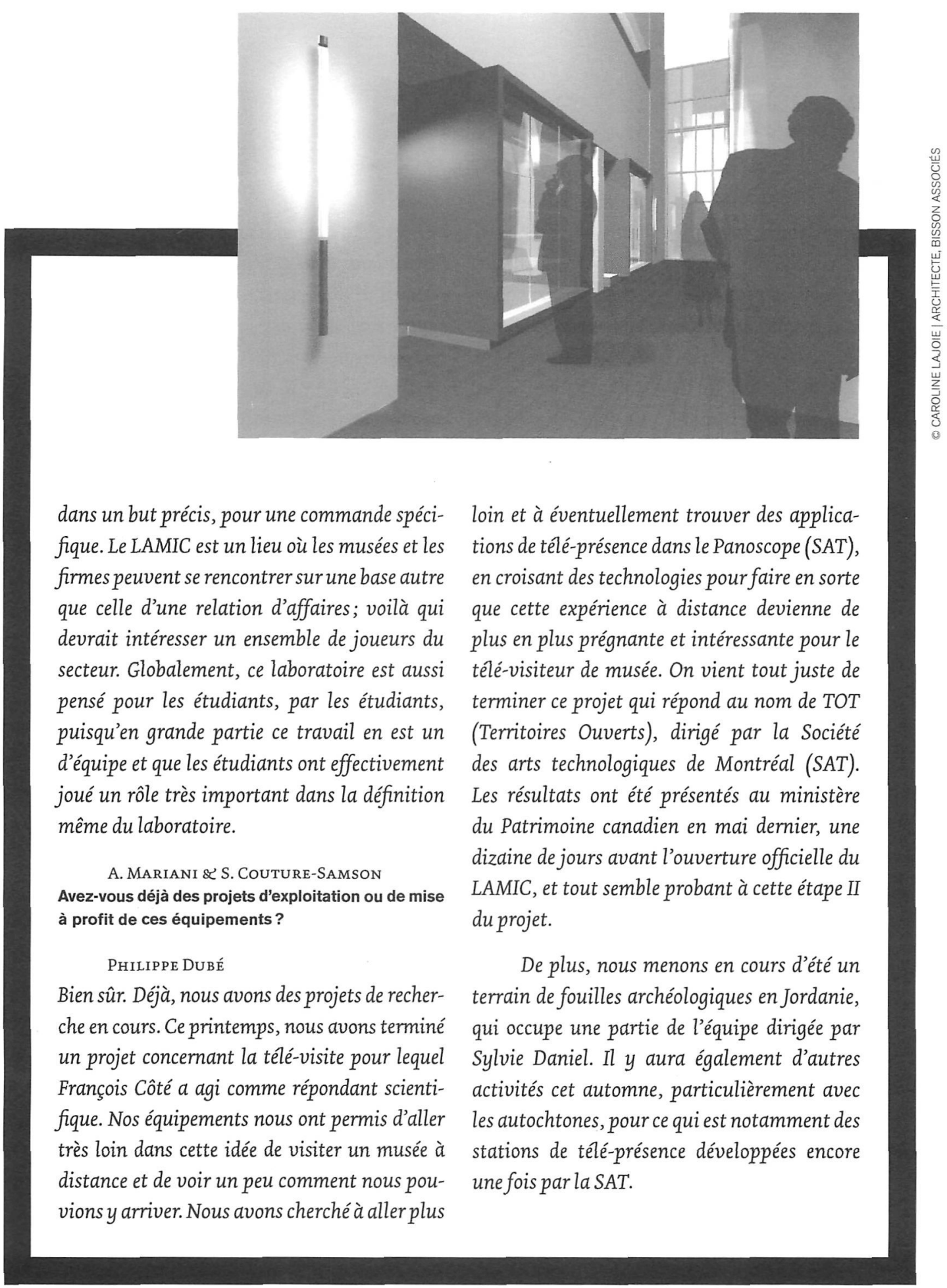


Bref, le LAMIC s'adresse à un public assez large, et aux étudiants d'abord et avant tout. Mais qu'est-ce que des étudiants de deuxième cycle peuvent faire avec ce laboratoire dans le programme du diplôme d'études supérieures spécialisées (DESS) tel qu'il est structuré présentement? C'est un problème dont il sera important de discuter dès cet automne en regard d'une réévaluation éventuelle du DESS en muséologie à l'Université Laval et ce, parce que nombre de projets font ressortir une contradiction patente : formation professionnelle versus recherche expérimentale (fondamentale et appliquée).

A. Mariani \& S. Couture-Samson Bien que l'ouverture du LAMIC soit récente, j'imagine que les musées ont déjà démontré de l'intérêt. Pouvez-vous élaborer sur l'éventualité de certains projets, de certaines collaborations?

\section{Philippe Dubé}

Le LAMIC s'adressant en grande partie aux étudiants, les travaux de certains doctorants qui ont commencé au mois d'août trouveront évidemment des applications directes au LAMIC. Mais ce ne sera pas le cas pour tous les étudiants. Par ailleurs, nous comptons surl'émulation et la contamination, pour éventuellement élargirle nombre d'étudiants intéressés à utiliser le laboratoire pour leurs propres travaux de recherche.

Nous avons également un réseau établi avec d'autres universités. Par exemple, nous pensons tisser des liens plus étroits avec le
MIT (Massachusetts Institute of Technology) et leur Medialab, qui nous intéresse beaucoup, en Belgique avec l'Université de Liège et en Allemagne, à Karlsruhe, avecle ZKM(Zentrum für Kunst und Medientechnologie), relié à une académie de beaux-arts très importante, où il se consacre à l'art technologique et aux médias electroniques. Il y fait des expérimentations fort importantes grâce à ce centre qui agit comme pôle international en la matière, et c'est évident que nous désirons nous joindre à eux, nous associer en quelque sorte à leur travail. Nous ne savons pas encore comment se matérialiseront ces ententes; tout cela se construit graduellement. Nous entretenons déjà des liens très étroits avec la SAT de Montréal, et celle-ci a son propre réseau, vaste et reconnu à travers le monde. Je crois beaucoup à la contamination. Ce qui est important, c'est qu'il se passe quelque chose et que ça ne tourne pas à vide ou en rond.

A. MARIANi \& S. COUTURE-SAMSON Quel aspect (exposition, diffusion, conservation, évaluation, recherche), selon vous, se porte présentement le mieux et pourquoi?

Philippe Dubé

Je vais répondre à cette question par son contraire. Ce qui va le mieux nous intéresse en fait plus ou moins. Par ailleurs ce qui m'intéresse davantage, c'est de voir ce qui va mal ou plutôt ce qui pose problème. Et, à mon sens, ce qui va le plus mal en notre domaine, c'est la recherche 
muséale. Et cela va jouer à la défaveur de ce qui s'en vient, car si nous ne nous attardons pas à la recherche, tout le reste risque fort d'en souffrir. L'exposition, la diffusion au sens large et même la conservation, pour laquelle il y a une avancée canadienne (l'Institut canadien de conservation y est pour beaucoup, mais aussi le CCQ/Centre de conservation $d u$ Québec) risquent de s'en voir perturbées. Là où le bât blesse, c'est vraiment surle plan de la recherche. Il est étonnant de voir dans quel état se trouvent des collections qui sont laissées à l'abandon, en termes de recherche. Et demain c'est l'exposition qui va en pâtir. Le musée n'est pas dans une position facile actuellement, je le reconnais. En fait, j'ai l'impression qu'il est condamné à se soumettre, étant à la solde des organismes subventionnaires culturels (et non de recherche) qui l'obligent à obéir à une logique du tourniquet imposant par là au musée de faire entrer du public à tout prix au lieu de poursuivre des études qui le nourrissent del'intérieur.

Pour moi, le médium exposition ne se prête pas nécessairement à une démonstration purement intellectuelle. L'exposition est le croisement de deux choses extrêmement importantes : l'émotion bien sûr, comme elément porteur, et la cognition comme elément de profondeur. C'est le croisement des deux qui fait que l'exposition prend sa pleine valeur. Il est clair que si la recherche se porte mal, c'est l'exposition qui souffrira d'une véritable mise à vide d'elle-même, le musée aussi par le fait même.

A. Mariani \& S. Couture-SAMson Tous savent que la recherche en muséologie est de plus en plus limitée et qu'elle est le plus souvent une affaire d'universitaires. Selon vous, de quelle façon la diffusion de ce savoir peut-elle le rendre plus accessible et, surtout, le mettre en valeur?

Philippe DubÉ

C'est une question à laquelle il est difficile de répondre. La recherche peut nous instruire essentiellement sur les modalités de transmission, c'est-à-dire sur le comment. Elle peut aussi nous donner des indications, par exemple penser que sur tel ou tel type de surface de plancher le visiteur est plus ou moins réceptif, ce qui peut favoriser ou non une meilleure transmission. Il ne s'agit là que d'un exemple d'ordre technique, mais qu'il est tout de même important de prendre en compte. Toutefois, ce qui est vraiment primordial, c'est de découvrir comment l'esprit humain fonctionne en contexte d'exposition, comment il réagit, à quoi il réagit, etc. Il est évident que ces deux aspects (technique et cognitif) font partie du programme général de la recherche qui se fera ici et qui devrait aider le musée à mieux transmettre effectivement. Vous avez remarqué que je préfere parler de transmission plutôt que de diffusion, parce que le musée n'est peut-être pas totalement adapté à l'aspect diffusion. Si l'on regarde les médias 
electroniques en général, la télévision particulièrement, quel magnifique médium pour diffuser. Mais, sur le plan de la transmission, sije vous demande ce que vous avez vu hier aux nouvelles télévisées, vous allez devoir faire un effort très important pour vous en rappeler, car la telévision reste un médium de diffusion très fluide, très efficace certes sur le plan de la propagation, mais qui, au volet de la transmission, est à peu près nul. Parce que ce qui nous en reste est le plus souvent maigre. Alors que le musée est a contrario un médium qui devrait offrir des expériences signifiantes. Je pense donc que la recherche qui sera réalisée ici devrait aider le musée à être un meilleur transmetteur. Mais de quelle façon, je ne le sais pas. Le temps, l'expérience et les travaux pourront seuls le dire. Laissons les choses se faire, les projets se réaliser, et nous verrons comment tout cela se développera. Chose certaine, le musée ne peut entreprendre à lui seul ce type de recherche et le relais est peut-être du côté universitaire. En fait, je n'y vois pas d'inconvénient majeur, carl'université est certainement plus indépendante (intellectuellement parlant) que ne l'est le musée. Et, de ce point de vue, les avancées en notre domaine risquent d'être plus grandes et ce sera au LAMIC de les diffuser sur son site Internet, qui devrait devenir une référence, principalement pour les petits et moyens musées (PMM).
A. MARIANI \& S. COUTURE-SAMSON Quels projets vous préoccupent présentement, mis à part le LAMIC?

\section{Philippe Dubé}

Précédemment, j'ai un peu vendu la mèche en mentionnant la muséalité en tant que pôle ou horizon de recherche, parce qu'au fond il s'agit d'une question très vaste, qui couvre tellement d'aspects, mais aussi qui peut mobiliser beaucoup d'énergie. Pour ce qui est du LAMIC, je crois que nous avons un horizon de recherche qui intéresse l'équipe en dehors du champ spécifique de leur investigation. Ce concept englobant me semble fédérateur au sein d'une équipe qui en couvre déjà large.

D'un point de vue plus personnel, je sais que je ne serai pas toujours à la barre du LAMIC, puisque ce n'est pas de cette manière que le laboratoire a été pensé. Un sujet qui attire mon attention de façon fondamentale est la question des PMM. J'espère pouvoir un joury revenir de façon sérieuse et travailler sur ce que j'ai déjà structuré. C'est-à-dire que j'ai déjà pensé à un petit traité de micro-muséologie; mon plan est fait, il s'agit simplement de le rédiger. C'est un projet sur lequel j'aimerais travailler de manière plus approfondie. Je ne sais pas encore très bien ce qui en sortira, mais je considère que c'est à ce chapitre précisément que j'ai le plus à dire. Peut-être est-ce quelque chose que je laisserai comme héritage. La micro-muséologie, telle que je la définis, et 
la question fondamentale du petit et moyen musée résument parfaitement bien l'ensemble de mes préoccupations.

La micro-muséologie est l'idée d'une muséologie qui concerne les petits organismes, des PMM et éventuellement des non-musées (le degré zéro du musée). Ce qui m'intéresse, c'est de partir d'une prémisse qui est d'affirmer que c'est là où se vivent les expériences muséales les plus signifiantes. Pour ma part, je les ai vécues dans des PMM. C'est un sujet d'étude auquel je veux revenir; je veux approfondir ces aspects. Permettez-moi de reprendre les mots de Régis Debray ${ }^{[3]}$ qui soutient qu'il n'y a de transmission possible qu'à la condition seule qu'il $y$ ait transformation. La diffusion, c'est essentiellement de l'information que l'on reçoit. Si une information est transmise, c'est qu'elle est venue nous transformer. C'est à cette considération profonde, je pense, que la micro-muséologie peut nous amener. Et les PMM, à cet égard, sont bien outillés, en termes de format notamment, pour faire ce genre de travail. J'aimerais vraiment pouvoir revenir éventuellement à ces recherches; il me semble que le musée ne peut se comprendre véritablement qu'à cette échelle.

[3]

DEBRAY, Régis.

Transmettre. Paris : Odile Jacob, 1997.
A. MARIANi \& S. Couture-SAmson Quel avenir entrevoyez-vous pour la muséologie? Quelles transformations sont à prévoir?

\section{Philippe Dubé}

$A u$ cours des vingt ou trente dernières années, le musée a franchi un pas très important, celui de l'interaction, de l'interactivité. En effet, pour une première fois, il a pris en compte ses publics, en réalisant qu'il ne pouvait plus être dans une relation autoritaire avec ces derniers. Il a commencé à considérer sérieusement le public et, éventuellement, est entré en relation avec lui. Cette question est, à mon avis, centrale pour parler $d u$ chemin accompli depuis les trente dernières années, dans les musées occidentaux plus particulièrement.

Selon moi, le prochain pas à franchir est, rapidement annoncé, l'immersion. Puisque le premier a été de briser la relation autoritaire qu'entretenait le musée envers ses publics, le prochain, la suite des choses, est de proposer au visiteur l'expérience englobante, complète, totale et intégrale. C'est le prochain pas à franchir pour vraiment offrir une expérience signifiante au visiteur. Nous serons passés de la visite simple de musée à l'expérience de musée et, en partie, ce passage, c'est d'abord l'interaction quil'aura permis. Et l'immersion, à mon sens, sera le deuxième pas qui va permettre d'offrir une expérience signifiante, globale, qui sera satisfaisante sur les plans à la fois cognitif, esthétique et émotif, vers une certaine intégralité de l'expérience de visite. 